\section{A associação entre a proximidade a equipamentos desportivos e a prática de atividade desportiva em adolescentes de 13 anos de idade do Município do Porto, Portugal}

\author{
The association between proximity to sports \\ facilities and participation in sports among \\ 13 -year-olds in the city of Porto, Portugal
}

\author{
1 Faculdade de Desporto, \\ Universidade do Porto, Porto, \\ Portugal. \\ 2 Instituto de Saúde Pública, \\ Universidade do Porto, Porto, \\ Portugal. \\ 3 Faculdade de Medicina, \\ Universidade do Porto, Porto, \\ Portugal. \\ 4 Instituto de Engenharia \\ Biomédica, Universidade do \\ Porto, Porto, Portugal. \\ Correspondência \\ E. Ramos \\ Instituto de Saúde Pública, \\ Universidade do Porto. \\ Rua das Taipas 135 , \\ Porto 4050-600, Portugal. \\ eliramos@med.up.pt
}

\begin{abstract}
The built environment can influence sports practice. The objective of this study was to examine the association between proximity $(\leq 500 \mathrm{~m})$ to sports facilities and participation in sports. We evaluated 1,175 13-year-olds (54\% girls) enrolled in public and private schools in Porto, Portugal. Sports activity was assessed using a self-completed questionnaire. The adolescents' residences and sports facilities were georeferenced. A matrix of distances from each residence to the facilities was calculated. For the majority (87\%) of the adolescents, there was at least one sports facility close to their place of residence. No statistically significant differences were found in participation in sports based on proximity to facilities $(\leq 500 \mathrm{~m}$ vs. > 500m); the proportions were $44 \%$ vs. $38 \%$ $(p=0.414)$ in girls and $72 \%$ vs. $61 \%(p=0.094)$ in boys. In conclusion, proximity to sports facilities was not significantly associated with participation in sports.
\end{abstract}

Sports Equipment; Sports; Adolescent

\author{
Roseanne Gomes Autran 1,2 \\ Elisabete Ramos 2,3 \\ Maria de Fátima de Pina 3,4 \\ Maria Paula Santos 1
}

\section{Introdução}

A prática da atividade física na adolescência proporciona vários efeitos benéficos à saúde, nomeadamente redução do risco de obesidade, melhoria da densidade mineral óssea e do perfil cardiovascular, quer a curto ou longo prazo 1,2 . Por outro lado, a prática da atividade física na adolescência parece aumentar a probabilidade do indivíduo tornar-se um adulto ativo ${ }^{3}$. Porém, apesar do conhecimento sobre os benefícios acerca da prática de atividade física, é elevada a proporção de adolescentes que não são suficientemente ativos ${ }^{4}$. Assim, é fundamental aumentar a prática de atividade física moderada - intensa, principalmente por meio da promoção da prática desportiva.

Para desenhar estratégias eficientes que atuem no aumento da atividade física e da prática desportiva é necessário conhecer os seus determinantes. Características individuais, sociais e demográficas (condição socioeconômica, sexo e idade), assim como a influência da família e dos amigos, têm sido identificadas na literatura 5,6, porém não têm sido suficientes para explicar as diferenças na prática.

A partir de 1990, houve um aumento relevante de estudos que abordavam atributos ambientais (segurança, acessibilidade, estética e disponibilidade de local para a prática desportiva) como fatores de influência na atividade física, e os resultados encontrados parecem in- 
dicar que estes atributos têm a capacidade de modificá-la 7,8,9,10.

No entanto, apesar da prática de desporto ser uma das componentes da atividade física avaliada nesses trabalhos, a informação sobre a relação entre a disponibilidade de equipamentos desportivos e prática de desporto é escassa, particularmente em adolescentes.

Foi observado nos adolescentes que a disponibilidade de equipamentos desportivos pode atuar como um facilitador para a mudança do comportamento em relação à prática desportiva, uma vez que aumenta a intenção da prática 11, mas não é claro se efetivamente a promove. Em Portugal, também se verificou uma associação positiva entre a percepção da existência de equipamentos e instalações desportivas com a prática de atividade física dos adolescentes 12,13,14.

A percepção que um indivíduo tem acerca das oportunidades para a prática de desporto na sua vizinhança pode divergir da informação objetiva sobre o que realmente existe. Tais diferenças podem estar relacionadas com o próprio indivíduo, por exemplo, um adolescente motivado para prática desportiva pode perceber mais oportunidades de prática na sua vizinhança do que um indivíduo menos motivado 15.

Nos últimos anos, houve um aumento no número de estudos que avaliavam o ambiente por meio de medidas objetivas. O uso de ferramentas tecnológicas como o Sistema de Informação Geográfica (SIG) é considerado superior em relação à avaliação perceptiva, pois permite a obtenção detalhada de informações acerca das oportunidades e disponibilidades existentes nas vizinhanças dos adolescentes que podem favorecer a prática desportiva 15 .

No entanto, não foram encontrados até a presente investigação, em Portugal, trabalhos que avaliassem de forma objetiva a proximidade a equipamentos desportivos e a sua associação com a prática desportiva, seja ela organizada ou não.

O presente estudo tem como objetivo avaliar a associação entre a proximidade a equipamentos desportivos na área de residência e a prática de atividades desportivas organizadas e/ou não organizadas relatadas por adolescentes de 13 anos de idade do Município do Porto, em Portugal.

\section{Metodologia}

\section{Área de estudo}

Segundo o Instituto Nacional de Estatística de Portugal (INE; http://www.ine.pt/xportal/
xmain?xpid=INE\&xpgid=ine_main), o Município do Porto tem uma área de $41,66 \mathrm{~km}^{2}$ e uma população de aproximadamente 210.558 habitantes (10.637.713 em Portugal), tratando-se de uma área urbana densamente povoada, com 5.099,8 habitantes por $\mathrm{km}^{2}$ (115,4 em Portugal). O Porto tem um clima atlântico temperado, apresentando uma temperatura média de $20,2^{\circ} \mathrm{C}$ em julho (verão) e de $9,3^{\circ} \mathrm{C}$ em janeiro (inverno). A precipitação média anual é de $1.253,5 \mathrm{~mm}$, sendo mais elevada nos meses de inverno.

\section{Participantes}

Este estudo foi desenvolvido no âmbito da coorte Epidemiological Health Investigation of Teenagers (EPITeen), iniciado em outubro de 2003 e concluída a la avaliação em julho de 2004, correspondendo a todo o ano letivo, com o objetivo de avaliar os adolescentes nascidos em 1990 que estudavam nas escolas públicas e privadas da cidade do Porto. Todas as escolas públicas (24) e 19 (79\%) privadas permitiram o contato com os alunos elegíveis e suas famílias. Nas escolas privadas que não aceitaram integrar o projeto, o número de adolescentes elegíveis era de aproximadamente 200. Na totalidade das escolas foram identificados 2.787 adolescentes elegíveis (2.126 nas públicas e 661 nas escolas privadas). Desses, 44 (1,6\%) nunca foram contatados por terem faltado às aulas durante o período de recrutamento, e 583 (20,9\%) não devolveram devidamente preenchido o documento para o consentimento informado, pelo que foram considerados recusas. Assim, participaram em algumas das etapas do projeto 2.160 (1.650 nas escolas públicas e 510 nas escolas privadas), sendo a proporção de participação de 77,5\%, idêntica nas escolas privadas $(77,7 \%)$ e nas públicas $(77 \%$, $\mathrm{p}=0,71)$. Segundo os dados da Direção Regional de Educação do Norte (DREN), essa distribuição por escolas públicas e privadas reflete a distribuição de alunos nas escolas do Município do Porto.

Dos 2.160 adolescentes avaliados, 1.398 eram elegíveis para este trabalho. Os restantes 762 adolescentes não foram considerados para esta investigação por morarem fora do Município do Porto e por não termos informações sobre as instalações desportivas existentes fora do município. Dos 1.398, foram excluídos da análise 216 $(18,8 \%)$ adolescentes que não responderam às questões sobre a prática de desporto, e sete por falta de informação que permitisse o georreferenciamento do local de residência. A amostra final do presente estudo foi de 1.175 adolescentes de 13 anos, dos quais 634 (54\%) meninas. Comparativamente com os adolescentes da amostra final, os adolescentes excluídos da análise têm uma proporção significativamente maior de meninos 
(56,5\% vs. $46 \%, \mathrm{p}=0,006)$, inscritos em escolas públicas $(88,4 \%$ vs. $80,4 \%, \mathrm{p}=0,007)$ e pais com menor escolaridade (média $=9,1$ anos; $\mathrm{DP}=4,6$ vs. média = 10,4 anos; $\mathrm{DP}=4,7 ; \mathrm{p}<0,001)$.

O projeto foi aprovado pela Comissão de Ética do Hospital de São João, e foi fornecida informação por escrito para os encarregados de educação. Obtivemos o consentimento informado dos adolescentes e dos encarregados de educação.

\section{Questionários}

Os dados sobre os adolescentes foram obtidos por meio da aplicação direta de dois questionários estruturados. Um foi enviado para casa e preenchido juntamente com os pais. O questionário preenchido em casa compreendia questões referentes a características sociais e demográficas (idade, escolaridade e profissão dos pais), comportamentais (hábito de fumar dos pais, hábitos alimentares e prática regular de desporto dos adolescentes) e de saúde (história clínica e utilização de cuidados de saúde) dos pais e dos adolescentes. Um segundo questionário foi preenchido pelo adolescente na escola, durante a visita da equipe de investigação, e compreendia as questões sobre o uso de álcool e cigarro.

Como indicador de classe social, utilizou-se a escolaridade dos progenitores, medida como o número máximo de anos completados com aproveitamento, tendo-se classificado o adolescente em função do progenitor mais escolarizado. Para classificar os hábitos tabágicos dos progenitores, como indicador de hábitos de saúde, foi utilizada a informação declarada pelo próprio aluno, considerando-se fumantes os pais que faziam uso do tabaco no período que correspondeu à avaliação.

\section{Atividade desportiva}

A informação sobre o desporto que o adolescente praticava e o tempo total gasto habitualmente por semana em cada desporto foi recolhida por meio de uma pergunta aberta, na qual o adolescente referia o desporto praticado (incluindo organizado e não organizado), a frequência por semana e o tempo que despendia com cada atividade. Para este estudo foi considerado a prática independentemente da frequência e tempo gasto em cada atividade. Consideramos prática de desporto apenas a prática fora das atividades escolares obrigatórias.

Também foi obtida a informação acerca do modo de deslocamento (ativo ou inativo) dos adolescentes para a escola. Considerou-se transporte ativo quando o adolescente referia ir a pé ou de bicicleta, e inativo se referia deslocar-se em veículo automotor (carro, moto, ônibus).

\section{Antropometria}

Na escola, uma equipe de profissionais de saúde realizou a avaliação do peso e altura com os adolescentes descalços e com roupas leves. Para a avaliação do peso foi utilizado um equipamento com bioimpedância (Tanita; Tanita Corp., Arlington Heights, Estados Unidos), com o participante sobre o centro da plataforma da balança com o peso distribuído igualmente pelos dois pés. A estatura foi medida com um estadiômetro portátil, o adolescente de pé, com os calcanhares unidos e a cabeça posicionada no plano horizontal de Frankfurt 16. Os adolescentes foram considerados com excesso de peso quando apresentavam índice de massa corporal (IMC) igual ou superior ao percentil 85, de acordo com o valor da distribuição em percentis para o sexo e a idade, elaborada pelos Centers for Disease Control and Prevention (CDC) dos Estados Unidos 17.

O IMC dos pais foi calculado com base no peso e altura autorrelatados e foram classificados em normal (IMC $<25,0 \mathrm{~kg} / \mathrm{m}^{2}$ ), excesso de peso (IMC $\geq 25,0 \mathrm{e}<30,0 \mathrm{~kg} / \mathrm{m}^{2}$ ) ou obesos (IMC $\left.\geq 30,0 \mathrm{~kg} / \mathrm{m}^{2}\right) 18$.

\section{Equipamentos desportivos}

Para este trabalho foram considerados apenas os espaços públicos de lazer. A lista desses equipamentos foi fornecida pela PortoLazer, órgão da Prefeitura Municipal responsável pelos equipamentos de lazer do município. Foram georreferenciados e considerados para este estudo, 89 espaços que possuíam equipamentos desportivos públicos, geridos pela PortoLazer. Os equipamentos que fizeram parte do estudo tiveram a sua estrutura física avaliada pelo mesmo observador. No âmbito desta avaliação o observador realizou uma classificação global do equipamento através de uma escala com quatro opções de respostas: insatisfatório, satisfatório, bom ou muito bom. Em entrevista aos responsáveis pelos equipamentos, foram registrados os tipos de modalidades oferecidas. Os dados foram recolhidos nos meses de outubro e novembro de 2009. Neste trabalho foram considerados apenas os equipamentos construídos antes de 2003.

\section{Georreferenciação}

As residências dos adolescentes foram georreferenciadas em ambiente de SIG, com base no endereço e utilizando um mapa digital da cidade do Porto contendo todos os trechos de ruas e intervalos de numeração, par e ímpar, de cada trecho. As instalações desportivas foram georreferenciadas com o uso do Sistema de Posicionamento Global 
(GPS), considerando a entrada exterior para as instalações.

A partir das coordenadas foram calculadas as distâncias euclidianas entre as residências dos adolescentes e os equipamentos desportivos, de maneira a gerar uma matriz de distâncias. Para cada estudante foi contabilizado o total de equipamentos de acordo com o estado de conservação e a variedade de modalidades disponíveis a uma distância $\leq 500 \mathrm{~m}$ das suas residências.

\section{Análise dos dados}

Para a análise estatística foi utilizado o programa SPSS, versão 16.0 (SPSS Inc., Chicago, Estados Unidos) e considerado um $\mathrm{p}<0,05$. Os resultados das variáveis quantitativas, referentes às distâncias para os equipamentos foram apresentados sob a forma de mediana (percentil 25-percentil 75). O teste de qui-quadrado $\left(\chi^{2}\right)$ foi usado para a comparação das proporções, e a associação entre a prática de desporto e as características dos equipamentos foi estimada por odds ratio (OR) e respectivos intervalos de 95\% de confiança (IC95\%) por meio da regressão logística não condicional com ajuste para a escolaridade dos pais.

\section{Resultados}

Dos 1.175 estudantes participantes deste trabalho, referiram praticar algum esporte 588 (49,8\%), com predominância dos meninos $(62,3 \%$ vs $39,2 \%, \mathrm{p}<0,001)$.

Nas meninas a prática de desporto teve maior frequência entre as que tinham pais com maior escolaridade, estavam inscritas em escolas privadas e que se deslocavam para a escola utilizando um meio de transporte inativo (Tabela 1). Nos rapazes apenas a escolaridade dos pais se associou

Fatores socioeconômicos e de tabaco relacionados com a prática de desporto.

\begin{tabular}{|c|c|c|c|c|c|c|}
\hline & \multicolumn{3}{|c|}{ Meninas } & \multicolumn{3}{|c|}{ Meninos } \\
\hline & \multicolumn{2}{|c|}{ Desporto } & \multirow{2}{*}{$\begin{array}{c}\text { Valor de } p \\
\qquad\left(\chi^{2}\right)\end{array}$} & \multicolumn{2}{|c|}{ Desporto } & \multirow{2}{*}{$\begin{array}{c}\text { Valor de } \mathrm{p} \\
\qquad\left(\chi^{2}\right)\end{array}$} \\
\hline & $\begin{array}{c}\text { Não pratica } \\
\text { (\%) }\end{array}$ & $\begin{array}{c}\text { Pratica } \\
\text { (\%) }\end{array}$ & & $\begin{array}{c}\text { Não Pratica } \\
(\%)\end{array}$ & $\begin{array}{c}\text { Pratica } \\
\text { (\%) }\end{array}$ & \\
\hline \multicolumn{7}{|l|}{ Escolaridade dos pais (anos) } \\
\hline$\leq 6$ & $147(78,2)$ & $41(21,8)$ & & $62(47,7)$ & $68(52,3)$ & \\
\hline $7-9$ & $95(68,3)$ & $44(31,7)$ & & $53(50,0)$ & $53(50,0)$ & $<0,001$ \\
\hline $10-12$ & $84(59,6)$ & $57(40,4)$ & $<0,001$ & $49(35,0)$ & $91(65,0)$ & \\
\hline$>12$ & $56(34,8)$ & $105(65,2)$ & & $38(23,9)$ & $121(76,1)$ & \\
\hline \multicolumn{7}{|l|}{ Tipo de escola } \\
\hline Pública & $325(65,0)$ & $175(35,0)$ & $<0,001$ & $70(38,2)$ & $275(61,8)$ & 0,839 \\
\hline Privada & $62(46,3)$ & $72(53,7)$ & & $35(36,5)$ & $61(63,5)$ & \\
\hline \multicolumn{7}{|l|}{ Transporte para a escola } \\
\hline Inativo & $143(52,0)$ & $132(48,0)$ & $<0,001$ & $95(39,4)$ & $146(60,6)$ & 0,909 \\
\hline Ativo & $205(70,0)$ & $88(30,0)$ & & $92(38,5)$ & $147(61,5)$ & \\
\hline \multicolumn{7}{|l|}{ IMC do adolescente * } \\
\hline$<$ percentil 85 & $265(58,4)$ & $189(41.6)$ & 0,158 & $146(37,1)$ & $248(62,9)$ & 0,861 \\
\hline$\geq$ percentil 85 e $<$ percentil 95 & $62(66,0)$ & $32(34,0)$ & & $30(40,0)$ & $45(60,0)$ & \\
\hline percentil $\geq 95$ & $36(69,2)$ & $16(30,8)$ & & $17(39,5)$ & $26(60,5)$ & \\
\hline \multicolumn{7}{|l|}{ IMC dos pais $\left(\mathrm{kg} / \mathrm{m}^{2}\right)$} \\
\hline$<25,0$ & $125(55,8)$ & $99(44,2)$ & & $59(37,8)$ & $97(62,2)$ & \\
\hline$\geq 25,0$ e $<30$ & $152(60,1)$ & $101(39,9)$ & 0,082 & $105(37,5)$ & $175(62,5)$ & 0,842 \\
\hline$\geq 30$ & $86(68,3)$ & $40(31,7)$ & & $27(34.2)$ & $52(68,8)$ & \\
\hline \multicolumn{7}{|l|}{ Fumantes (pais) } \\
\hline Não & $52(60,5)$ & $34(39,5)$ & 1,000 & $37(33,6)$ & $73(66,4)$ & 0,861 \\
\hline Sim & $312(60,0)$ & $208(40,0)$ & & $153(37,4)$ & $323(62,6)$ & \\
\hline
\end{tabular}

IMC: índice de massa corporal.

* Classificação do Centers for Disease Control and Prevention (CDC) 17: normal (< percentil 85); sobrepeso ( $\geq$ percentil 85 e

$<$ percentil 95) e obeso (percentil $\geq 95$ ). 
significativamente com a prática de desporto (Tabela1).

Os adolescentes possuíam em média (desviopadrão - DP) $2(1,4)$ equipamentos, sendo que $12,6 \%$ dos estudantes não possuíam equipamentos desportivos na proximidade das suas residências. Quando avaliado o estado de conservação dos equipamentos, $47 \%$ dos adolescentes não possuíam equipamentos com o estado de conservação "muito bom" ou "bom", e 22,7\% deles possuíam apenas um equipamento com o estado de conservação “muito bom” ou “bom”. Em relação à oferta na variedade de modalidades desportivas, verificou-se que a modalidade mais frequentemente disponível era futebol e/ou futsal $(39,9 \%)$, seguida de basquete $(22,3 \%)$ e voleibol $(7,4 \%)$. Tendo em conta a distância da residência às diferentes modalidades, verificamos que $22,1 \%$ dos adolescentes possuíam 1 modalidade desportiva e 35\% deles possuíam de 2 a 3 modalidades diferentes numa distância de até $500 \mathrm{~m}$. Não se observaram diferenças estatisticamente significativas entre os que praticavam e os que não praticavam desporto quando considerada a distância ao equipa- mento mais próximo do local de residência. Para as meninas a mediana (percentil 25-percentil 75) da distância (em metros) para o equipamento mais próximo era 278,0 $(158,1-396,2)$ entre as que não praticavam desporto e 308,6 (164,6-439,5) entre as que praticavam $(\mathrm{p}=0,082)$. Nos meninos esses valores correspondem a 281,6 (152,2-401,8) e 285,5 (172,4-427,5), respectivamente, para os que não praticavam e para os que praticavam desporto ( $\mathrm{p}=0,265)$.

Quando estratificado por gênero, nas meninas (Tabela 2) nenhuma das características do equipamento se associou significativamente com a prática desportiva, verificamos apenas que as meninas com 4 ou mais equipamentos praticavam significativamente menos desportos (OR $=0,48$, IC95\%: 0,24-0,96). Nos meninos verificou-se que os adolescentes que tinham oferta de 4 ou mais modalidades apresentaram um OR de 1,82 (IC95\%: 1,122,98) de praticar desporto relativamente aos que tinham apenas uma modalidade (Tabela 3). Em ambos os gêneros, os valores de OR permaneceram semelhantes após ajuste para a escolaridade dos pais (Tabelas 2 e 3 ).

Características dos equipamentos desportivos relacionados com a prática de desporto referente ao gênero feminino.

\begin{tabular}{|c|c|c|c|c|c|}
\hline \multirow[t]{2}{*}{ Distância $\geq 500 m$} & \multicolumn{3}{|c|}{ Desporto } & \multicolumn{2}{|c|}{ Desporto } \\
\hline & $\begin{array}{c}\text { Não pratica } \\
\text { (\%) }\end{array}$ & $\begin{array}{c}\text { Pratica } \\
\text { (\%) }\end{array}$ & Valor de $p\left(\chi^{2}\right)$ & $\begin{array}{l}\text { OR bruto } \\
\text { (IC95\%) }\end{array}$ & $\begin{array}{l}\text { OR ajustado * } \\
\text { (IC95\%) }\end{array}$ \\
\hline \multicolumn{6}{|l|}{ Possui equipamento } \\
\hline Não & $19(27,9)$ & $49(72,1)$ & & Referência & Referência \\
\hline Sim & $186(39,3)$ & $287(60,7)$ & 0,094 & $0,59(0,34-1,03)$ & $0,61(0,34-1,10)$ \\
\hline \multicolumn{6}{|c|}{ Número de equipamentos com estado } \\
\hline \multicolumn{6}{|c|}{ de conservação (bom e muito bom) } \\
\hline 0 & $101(38,8)$ & $159(61,2)$ & & Referência & Referência \\
\hline 1 & $60(39,2)$ & $93(60,8)$ & 0,642 & $0,99(0,65-1,48)$ & $1,09(0,71-1,66)$ \\
\hline$\geq 2$ & $44(34,4)$ & $84(65,6)$ & & $1,22(0,78-1,90)$ & $1,20(0,76-1,90)$ \\
\hline \multicolumn{6}{|c|}{ Número de equipamentos } \\
\hline 0 & $19(27,9)$ & $49(72,1)$ & & Referência & Referência \\
\hline 1 & $68(41,0)$ & $98(59,0)$ & 0,318 & $0,55(0,30-1,01)$ & $0,55(0,29-1,04)$ \\
\hline 2 & $57(41,6)$ & $80(58,4)$ & & $0,54(0,29-1,01)$ & $0,53(0,27-1,01)$ \\
\hline 3 & $36(35,0)$ & $67(65,0)$ & & $0,71(0,36-1,38)$ & $0,71(0,36-1,43)$ \\
\hline$\geq 4$ & $25(37,3)$ & $42(62,7)$ & & $0,65(0,32-1,35)$ & $0,86(0,41-1,84)$ \\
\hline \multicolumn{6}{|c|}{ Total de modalidades ofertadas } \\
\hline 1 & $58(42,2)$ & $65(52,8)$ & 0,067 & Referência & Referência \\
\hline 2 & $37(43,0)$ & $49(57,0)$ & & $1,18(0,68-2,06)$ & $1,14(0,64-2,04)$ \\
\hline 3 & $34(37,4)$ & $57(62,6)$ & & $1,50(0,86-2,60)$ & $1,46(0,82-2,58)$ \\
\hline$\geq 4$ & $57(32,6)$ & $118(67,4)$ & & $1,85(1,15-2,97)$ & $1,82(1,12-2,98)$ \\
\hline
\end{tabular}

OR: odds ratio.

* OR ajustado para a escolaridade dos pais. 
Características dos equipamentos desportivos relacionados com a prática de desporto referente ao gênero masculino.

\begin{tabular}{|c|c|c|c|c|c|}
\hline \multirow[t]{2}{*}{ Distância $\geq 500 \mathrm{~m}$} & \multicolumn{3}{|c|}{ Desporto } & \multicolumn{2}{|c|}{ Desporto } \\
\hline & Não pratica (\%) & Pratica (\%) & Valor de $p\left(\chi^{2}\right)$ & OR bruto (IC95\%) & $\begin{array}{l}\text { OR ajustado * } \\
\text { (IC95\%) }\end{array}$ \\
\hline \multicolumn{6}{|l|}{ Possui equipamento } \\
\hline Não & $19(27,9)$ & $49(72,1)$ & & Referência & Referência \\
\hline Sim & $186(39,3)$ & $287(60,7)$ & 0,094 & $0,59(0,34-1,03)$ & $0,61(0,34-1,10)$ \\
\hline \multicolumn{6}{|c|}{ Número de equipamentos com estado } \\
\hline \multicolumn{6}{|c|}{ de conservação (bom e muito bom) } \\
\hline 0 & $101(38,8)$ & $159(61,2)$ & & Referência & Referência \\
\hline 1 & $60(39,2)$ & $93(60,8)$ & 0,642 & $0,99(0,65-1,48)$ & $1,09(0,71-1,66)$ \\
\hline$\geq 2$ & $44(34,4)$ & $84(65,6)$ & & $1,22(0,78-1,90)$ & $1,20(0,76-1,90)$ \\
\hline \multicolumn{6}{|c|}{ Número de equipamentos } \\
\hline 0 & $19(27,9)$ & $49(72,1)$ & & Referência & Referência \\
\hline 1 & $68(41,0)$ & $98(59,0)$ & 0,318 & $0,55(0,30-1,01)$ & $0,55(0,29-1,04)$ \\
\hline 2 & $57(41,6)$ & $80(58,4)$ & & $0,54(0,29-1,01)$ & $0,53(0,27-1,01)$ \\
\hline 3 & $36(35,0)$ & $67(65,0)$ & & $0,71(0,36-1,38)$ & $0,71(0,36-1,43)$ \\
\hline$\geq 4$ & $25(37,3)$ & $42(62,7)$ & & $0,65(0,32-1,35)$ & $0,86(0,41-1,84)$ \\
\hline \multicolumn{6}{|c|}{ Total de modalidades ofertadas } \\
\hline 1 & $58(42,2)$ & $65(52,8)$ & 0,067 & Referência & Referência \\
\hline 2 & $37(43,0)$ & $49(57,0)$ & & $1,18(0,68-2,06)$ & $1,14(0,64-2,04)$ \\
\hline 3 & $34(37,4)$ & $57(62,6)$ & & $1,50(0,86-2,60)$ & $1,46(0,82-2,58)$ \\
\hline$\geq 4$ & $57(32,6)$ & $118(67,4)$ & & $1,85(1,15-2,97)$ & $1,82(1,12-2,98)$ \\
\hline
\end{tabular}

OR: odds ratio.

* OR ajustado para a escolaridade dos pais.

Considerando as modalidades referidas pelos estudantes que praticavam algum desporto, as mais praticadas pelas meninas eram natação $(23,5 \%)$, dança e/ou balé $(20,1 \%)$ e fitness $(10,2 \%)$. Entre os meninos, as modalidades desportivas mais referidas foram futebol e/ou futsal $(36,7 \%)$, natação (16\%) e tênis (10,5\%). Levando em conta a distribuição da oferta dessas modalidades em função do local de residência, verificamos que $70,1 \%$ dos meninos tinham alguma das 3 modalidades que mais referiram praticar a uma distância $\leq 500 \mathrm{~m}$ da sua residência. Entre as meninas apenas $17,2 \%$ tinham a essa distância pelo menos um equipamento que oferecia alguma das modalidades que mais referiram praticar (Figura 1). Nenhum equipamento oferecia a prática de fitness, apenas um oferecia balé e cinco ofereciam natação, sendo estas inversamente as três modalidades mais referidas pelas meninas. Quando analisada a relação entre as modalidades desportivas ofertadas num raio $\leq 500 \mathrm{~m}$ e as referidas pelos adolescentes, observou-se que $78,5 \%$ das meninas que praticavam alguma modalidade não praticavam nenhuma das existentes na proximidade da sua residência.

\section{Discussão}

Este estudo destinou-se a analisar a associação entre a proximidade a equipamentos desportivos, avaliados de forma objetiva, e a prática de esportes autorrelatada em uma amostra de adolescentes de 13 anos de idade do Município do Porto. Não foi encontrada associação significativa entre a presença de equipamentos desportivos num raio $\leq 500 \mathrm{~m}$ das residências e a prática desportiva autorreportada pelos estudantes de ambos os sexos.

Esses resultados estão em concordância com os encontrados numa amostra de adolescentes holandeses 15 e em adolescentes de 14 anos de idade da Nova Zelândia 2 a 95\%, nos quais não se encontrou uma associação significativa entre a existência de instalações desportivas e a prática de desporto. Assim, embora a presença de instalações desportivas possa ser considerada facilitadora para o desenvolvimento da prática, pode não ser suficiente para que os indivíduos modifiquem o seu comportamento de não praticantes para praticantes 20 . 
Figura 1

Distribuição da oferta de modalidades desportivas mais praticadas por gênero.

1a) Meninos

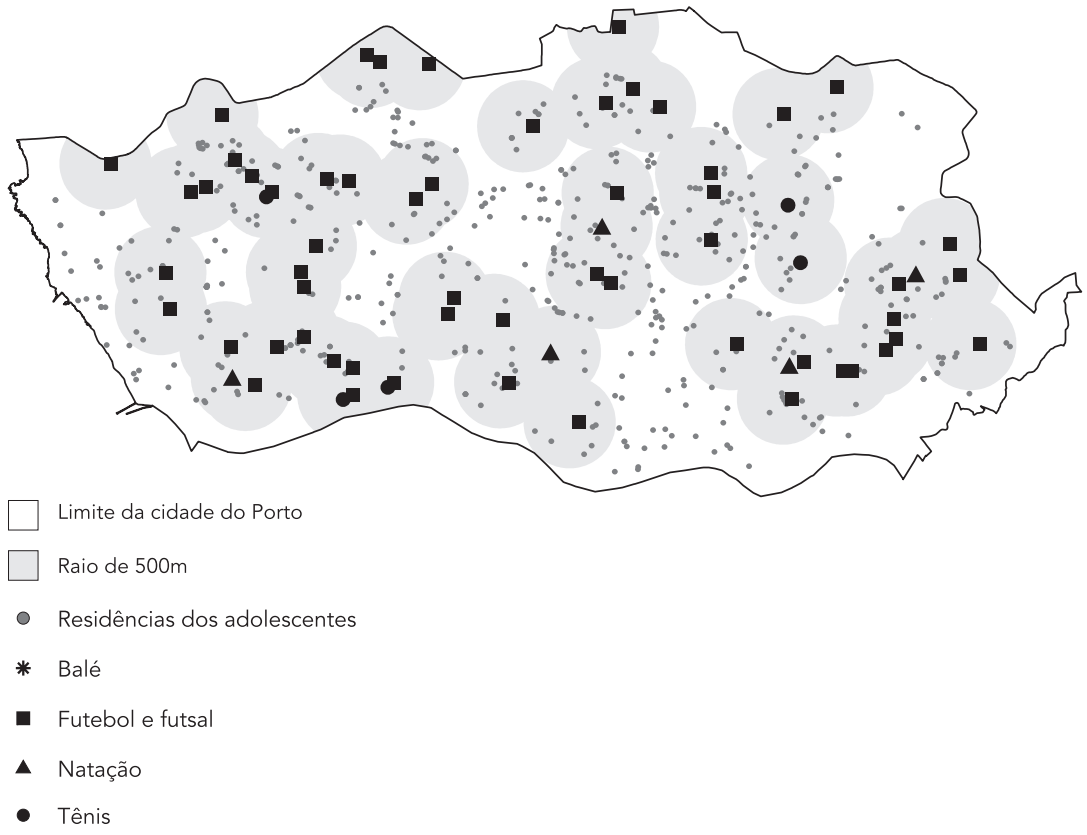

1b) Meninas

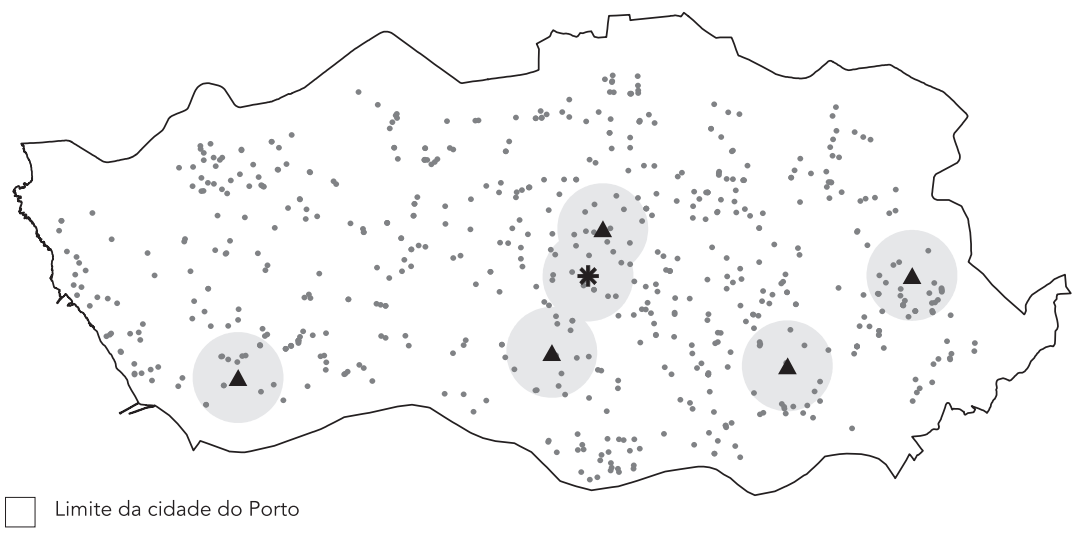

Raio de $500 \mathrm{~m}$

- Residências dos adolescentes

* Balé

- Futebol e futsal


\ Natação

- Tênis

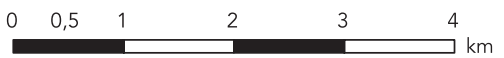


Por outro lado, também as condições de conservação e de acesso podem condicionar a utilização dos equipamentos 13. No entanto, no nosso trabalho, o estado de conservação dos equipamentos existentes nas proximidades das residências também não se associou com a prática de desporto. Esses resultados parecem contrariar os encontrados em outros estudos numa população de adolescentes, na qual se verificou uma menor prática desportiva quando estes avaliavam o local como sendo "feio" ou "carente de instalações noturnas" 14. É importante realçar que no nosso estudo a avaliação do estado de conservação dos equipamentos foi feita pelo investigador, enquanto no estudo anterior é medida a percepção do adolescente, o que pode justificar a diferença de resultados.

Outro aspecto a ser considerado é que a condição socioeconômica e o contexto escolar podem ser determinantes fundamentais para a prática desportiva entre estes adolescentes, no entanto, apenas foram avaliados os espaços públicos para a prática desportiva.

Nos meninos, verificamos um aumento da proporção de praticantes de desporto em função da maior variedade de modalidades desportivas disponíveis numa distância de $\leq 500 \mathrm{~m}$ da sua residência, entretanto, este efeito não foi significativo. Também para as meninas não foi encontrada associação positiva. Apesar disso, há significativa desigualdade entre gêneros no que diz respeito à oferta de variedade de modalidades, sendo que $70 \%$ dos meninos possuíam na proximidade de suas residências pelo menos uma das modalidades desportivas por eles preferidas, enquanto para as meninas apenas $17 \%$ estavam nestas condições. Os nossos dados mostram uma associação inversa entre o número de equipamentos e a prática de desportes pelas meninas, o que pode ser justificado porque os equipamentos disponibilizados são essencialmente para desportos culturalmente mais praticados pelos meninos (futebol e futsal). Esse resultado é importante na medida em que a variedade na oferta de modalidades desportivas é essencial para que os espaços se tornem mais atrativos 3, e o fácil acesso às modalidades desportivas pode permitir uma maior adesão à prática do desporto 11.

O fato de não conhecermos os motivos de escolha da modalidade desportiva, as percepções dos adolescentes sobre a disponibilidade de equipamentos ou os motivos da escolha do local de prática esportiva limita a interpretação dos resultados obtidos. Por outro lado, o trabalho apresenta como forças relevantes o tamanho da amostra, o fato de ocorrer numa idade em que o ensino é obrigatório em Portugal, tornando a escola uma excelente base amostral, e a proporção de participação no estudo ser elevada. É de realçar o fato de terem sido avaliados alunos dos ensinos público e privado, uma vez que estudos anteriores mostraram que os fatores socioeconômicos são determinantes fundamentais da prática de desporto 5,6 . Não podemos omitir que as diferenças entre os participantes e os excluídos condicionaram menor representatividade dos meninos e dos adolescentes com condição socioeconômica menor, isto promove uma menor variabilidade entre os participantes e pode ter contribuído para esbater uma possível associação entre as características do equipamento e a prática desportiva.

Os resultados encontrados nesta investigação podem contribuir para o planejamento de estratégias que visem ao aumento da prática de desporto. A variedade na oferta de modalidades desportivas é essencial para que os espaços tornem-se atrativos 21 . Porém, a construção de equipamentos pode não ser uma estratégia adequada particularmente na relação custo benefício, a diversificação da oferta de modalidades nos equipamentos já existentes e a adequação desta oferta às preferências dos adolescentes poderá, com menor custo, facilitar o aumento da prática nesta população. No entanto, para além desses dados é relevante a avaliação de outros fatores determinantes da atividade desportiva dos adolescentes que possam mediar a associação entre a proximidade a equipamentos e a prática desportiva, como por exemplo, a condição socioeconômica, a acessibilidade às instalações esportivas e a segurança.

\section{Conclusão}

A proximidade de equipamentos desportivos ao local de residência e o seu estado de conservação não se associou com a prática de atividade desportiva entre os adolescentes do Porto. No entanto, um maior número de modalidades ofertadas parece favorecer a prática de desporto.

É importante para além das variáveis ambientais abordadas neste estudo, investigarmos que tipos de comportamentos os adolescentes adotam fora do âmbito escolar e qual a relação deste comportamento com a adesão à prática desporto nos tempos livres. 


\section{Resumo}

O ambiente construído pode influenciar a prática desportiva. O objetivo foi examinar a associação entre a proximidade (500m) de equipamentos desportivos e a participação em atividades desportivas. Foram avaliados 1.175 (54\% meninas) estudantes de 13 anos de idade matriculados em escolas públicas e privadas do Município do Porto, Portugal. A atividade desportiva foi avaliada utilizando um questionário autoaplicável. As residências dos participantes e os equipamentos públicos foram georreferenciados. Foi calculada uma matriz de distância da residência de cada participante até os equipamentos. A maioria dos adolescentes (87\%) reside na proximidade de, pelo menos, um equipamento desportivo. Não foram encontradas diferenças na prática desportiva relacionadas com a proximidade aos equipamentos ( $\leq 500 \mathrm{~m}$ vs. $>500 \mathrm{~m}$ ), sendo as proporções $44 \%$ vs. $38 \%, p=0.414$ (meninas) e $72 \%$ vs. $61 \%, p=0.094$ (meninos). A proximidade de equipamentos não estava associada com a prática do desporto.

Equipamentos Esportivos; Esportes; Adolescente

\section{Colaboradores}

R. G. Autran participou da análise e interpretação dos dados, redação do artigo e aprovação final da versão a ser publicada. E. Ramos e M. F. Pina colaboraram na análise e interpretação dos dados, revisão crítica relevante do conteúdo intelectual e aprovação final da versão a ser publicada. M. P. Santos contribuiu na revisão crítica relevante do conteúdo intelectual e aprovação da versão final.

\section{Agradecimentos}

Este trabalho foi desenvolvido no âmbito dos Projetos Pluridisciplinares de Iniciação à Investigação da Universidade do Porto (concurso 2009). Os autores agradecem também à Fundação para a Ciência e Tecnologia pelo financiamento concedido ao projeto EPITeen (POCTI/SAU-ESP/62399/2004).

\section{Referências}

1. Pitukcheewanont P, Punyasavatsut N, Feuille M. Physical activity and bone health in children and adolescents. Pediatr Endocrinol Rev 2010; 7 : 275-82.

2. Steele RM, Brage S, Corder K, Wareham NJ, Ekelund U. Physical activity, cardiorespiratory fitness, and the metabolic syndrome in youth. J Appl Physiol 2008; 105:342-51.

3. Telama R, Yang X, Viikari J, Välimäki I, Wanne O, Raitakari O. Physical activity from childhood to adulthood: a 21-year tracking study. Am J Med 2005; 28:267-73.

4. Hills AP, King NA, Armstrong TP. The contribution of physical activity and sedentary behaviours to the growth and development of children and adolescents: implications for overweight and obesity. Sports Med 2007; 37:533-45.

5. Toftegaard-Støckel J, Nielsen GA, Ibsen B, Andersen LB. Parental, socio and cultural factors associated with adolescents' sports participation in four Danish municipalities. Scand J Med Sci Sports 2011;21:606-11.
6. Seabra AE, Mendonça DM, Thomis MA, Anjos LA, Maia JA. Determinantes biológicos e sócio-culturais associados à pratica de atividade física de adolescentes. Cad Saúde Pública 2008; 24:721-36.

7. Hoehner CM, Brennan Ramirez LK, Elliott MB, Handy SL, Brownson RC. Perceived and objective environmental measures and physical activity among urban adults. Am J Prev Med 2005; 28(2 Suppl 2): 105-16.

8. Boehmer TK, Lovegreen SL, Haire-Joshu D, Brownson RC. What constitutes an obesogenic environment in rural communities? Am J Health Promot 2006; 20:411-21.

9. Salmon J, Timperio A. Prevalence, trends and environmental influences on child and youth physical activity. Med Sports Sci 2007; 50:183-99.

10. Sallis JF. Measuring physical activity environments: a brief history. Am J Prev Med 2009; 36(4 Suppl):S86-92. 
11. Prins RG, van Empelen P, Te Velde SJ, Timperio A, van Lenthe FJ, Tak NI, et al. Availability of sports facilities as moderator of the intention-sports participation relationship among adolescents. Health Educ Res 2010; 25:489-97.

12. Mota J, Almeida M, Santos MP, Ribeiro JC. Perceived neighborhood environments and physical activity in adolescents. Prev Med 2005; 41:834-6.

13. Santos MP, Page AS, Cooper AR, Ribeiro JC, Mota J. Perceptions of the built environment in relation to physical activity in Portuguese adolescents. Health Place 2009; 15:548-52.

14. Loureiro NE, Matos MG, Santos MM, Mota JA, Diniz JA. Neighborhood and physical activities of Portuguese adolescents. Int J Behav Nutr Phys Act 2010; 7:33.

15. Prins RG, Oenema A, van der Horst K, Brug J. Objective and perceived availability of physical activity opportunities: differences in associations with physical activity behavior among urban adolescents. Int J Behav Nutr Phys Act 2009; 6:70.

16. World Health Organization. Physical status: the use interpretation of anthropometry. Report of a WHO Expert Committee. Geneva: World Health Organization; 1995. (WHO Technical Report Series, 854).
17. Kuczmarski RJ, Ogden CL, Guo SS, GrummerStrawn LM, Flegal KM, Mei Z, et al. 2000 CDC Growth Charts for the United States: methods and development. Vital Health Stat 11 2002; (246): 1-190.

18. Clinical guidelines on the identification, evaluation, and treatment of overweight and obesity in adults: executive summary. Expert Panel on the Identification, Evaluation, and Treatment of Overweight in Adults. Am J Clin Nutr 1998; 68:899-917.

19. Maddison R, Hoorn SV, Jiang Y, Mhurchu CN, Exeter D, Dorey E, et al. The environment and physical activity: the influence of psychosocial, perceived and built environmental factors. Int J Behav Nutr Phys Act 2009; 6:19.

20. Giles-Corti B, Donovan RJ. Increasing walking: relative influences of individual, social environmental, and physical environmental correlates of walking. Am J Public Health 2003; 93:1583-9.

21. Santos MP, Gomes H, Mota J. Physical activity and behaviors sedentary in adolescents. Ann Behav Med 2005; 30:21-4.

Recebido em 18/Fev/2011

Versão final reapresentada em 23/Set/2011

Aprovado em 20/Out/2011 